This is the peer-reviewed version of the article:

Marko Perić, Amador Garcia-Fuente, Matija Zlatar, Claude Daul, Stepan Stepanović, Pablo Garcia-Fernndez, Maja Gruden-Pavlović, Magnetic Anisotropy in "Scorpionate" First-Row Transition-Metal Complexes: A Theoretical Investigation, Chemistry-A European Journal, 2015, 21, 9, 3716-3726, https://dx.doi.org/10.1002/chem.201405480 


\title{
Magnetic Anisotropy in "Scorpionate" First-Row Transition-Metal Complexes: A Theoretical Investigation
}

\author{
Marko Perić, ${ }^{[[a]}$ Amador García-Fuente, ${ }^{[b]}$ Matija Zlatar, ${ }^{[a]}$ Claude Daul $_{,}^{[b]}$ Stepan Stepanović, ${ }^{[a]}$ \\ Pablo García-Fernández, ${ }^{[c]}$ and Maja Gruden-Pavlović ${ }^{*[d]}$
}

\begin{abstract}
In this work we have analyzed in detail the magnetic anisotropy in a series of hydrotris(pyrazolyl)borate $\left(\mathrm{Tp}^{-}\right)$metal complexes, namely $[\mathrm{VTpCl}]^{+},[\mathrm{CrTpCl}]^{+}$, $[\mathrm{MnTpCl}]^{+},[\mathrm{FeTpCl}],[\mathrm{CoTpCl}]$, and $[\mathrm{NiTpCl}]$, and their substituted methyl and tert-butyl analogues with the goal of observing the effect of the ligand field on the magnetic properties. In the $[\mathrm{VTpCl}]^{+},[\mathrm{CrTpCl}]^{+},[\mathrm{CoTpCl}]$, and $[\mathrm{NiTpCl}]$ complexes, the magnetic anisotropy arises as a consequence of out-of-state spin-orbit coupling, and covalent changes induced by the substitution of hydrogen atoms on the pyrazolyl rings does not lead to drastic changes in the magnetic anisotropy. On the other hand, much larger magnetic anisotropies were predicted in complexes displaying a degenerate ground state, namely $[\mathrm{MnTpCl}]^{+}$and $[\mathrm{FeTpCl}]$, due to instate spin-orbit coupling. The anisotropy in these systems
\end{abstract}

\section{Introduction}

The analysis of the origin of magnetic interactions in complexes with $d$ - and f-metal ions has become the subject of extensive research in the past few years, and the theoretical aspects have been described in many books and papers. ${ }^{[1]}$ Detailed analyses and further explanations of magnetic properties at the molecular level could give a deeper insight into the characteristics of magnetization at the macroscopic level.

One of the most interesting discoveries has been the slow relaxation of magnetization at the molecular level. ${ }^{[2]}$ This prop-

[a] Dr. M. Perić, Dr. M. Zlatar, S. Stepanović

Center for Chemistry, Institute of Chemistry

Technology and Metallurgy, University of Belgrade

Njegoseva 12, 11001 Belgrade (Serbia)

E-mail:markop@chem.bg.ac.rs

[b] Dr. A. García-Fuente, Prof. C. Daul

Department of Chemistry, University of Fribourg

Chemin du Musée 9, 1700 Fribourg (Switzerland)

[c] Dr. P. García-Fernández

Ciencias de la Tierra y Física de la Materia Condensada

Universidad de Cantabria, Santander (Spain)

[d] Prof. M. Gruden-Pavlović

Faculty of Chemistry, University of Belgrade

Studentski trg 12-16, 11001 Belgrade (Serbia)

E-mail:gmaja@chem.bg.ac.rs

Supporting information for this article is available was shown to be very sensitive to perturbations, for example, chemical substitution and distortions due to the JahnTeller effect. We found that by substituting the hydrogen atoms in $[\mathrm{MnTpCl}]^{+}$and $[\mathrm{FeTpCl}]$ by methyl and tert-butyl groups, certain covalent contributions to the magnetic anisotropy energy (MAE) could be controlled, thereby achieving higher values. Moreover, we showed that the selection of ion has important consequences for the symmetry of the ground spin-orbit term, opening the possibility of achieving zero magnetic tunneling even in non-Kramers ions. We have also shown that substitution may also contribute to a quenching of the Jahn-Teller effect, which could significantly reduce the magnetic anisotropy of the complexes studied.

erty strongly contrasts with that found in conventional bulk magnets, in which the magnetization decay is directly linked to the particle size, becoming faster as the particle size is reduced. Systems of this kind are called single-molecule magnets $(\mathrm{SMMs})^{[3]}$ and are very interesting candidates for high-density data storage ${ }^{[4]}$ as well as for the development of quantum computers. $^{[2 \mathrm{~b}]}$

The basic requirement necessary for a long relaxation time is the existence of an energy barrier that separates states with the same magnitude of magnetic moment but opposite directions. In other words, magnetic anisotropy manifests itself as an energy barrier that spin vectors must overcome when they switch from one direction to the other. For systems with axial symmetry, the energy of this barrier is usually described by Equation (1), in which $U_{\text {eff }}$ represents the effective barrier, $D$ represents the axial zero-field splitting (ZFS) parameter, and $S_{\max }$ is the total spin of the molecule.

$U_{\text {eff }}=|D| S_{\max }^{2}$

$U_{\text {eff }}$ has been found to be largely independent of the total spin as $D$ is inversely proportional to $S^{2} .{ }^{[5]}$ The axial parameter $D$ is a measure of the magnetic anisotropy energy (MAE) of a complex, that is, the higher is the absolute value of the axial parameter, the greater will be the MAE of the system. For Equation (1) to represent a barrier between two stable magnetic states, as required for most technological applications, $D$ 
must be negative. ${ }^{[6]}$ However, for a half-integer spin ground state, even positive $D$ values are found to show SMM behavior $^{[5 a]}$ because magnetic tunneling is exactly zero due to symmetry arguments. The MAE in transition-metal complexes is still not well understood, particularly its link with the geometry of the system, and significant effort has been made ${ }^{[7]}$ to clarify this situation as such knowledge would probably facilitate the discovery of new systems with suitable properties for application.

Computational simulations have been found to be a very useful tool for understanding and predicting the magnetic properties of $\mathrm{d}$ - and $\mathrm{f}$-metal complexes. ${ }^{[8]}$ Of all the different existing quantum mechanical methods, the Kohn-Sham density functional theory (KS-DFT) ${ }^{[9]}$ is especially relevant in the simulation of large systems, including tens of atoms, due to the compromise offered between accuracy and computational cost. However, it is essential to mention that DFT techniques based on perturbation theory may give very poor results if they are employed for the analysis of the MAE in transitionmetal complexes. ${ }^{[7 a]}$ On the other hand, highly correlated wavefunction-based methods provide, in principle, accurate results, but they are computationally very expensive. A very reasonable compromise may be found by using a multideterminant DFT-based method combined with ligand-field theory (LF-DFT), which has been shown to perform remarkably well for calculations of MAEs. ${ }^{[10]}$ The LFD-FT model ${ }^{[10 b, c]}$ is based on a multideterminant description of the multiplet structures ${ }^{[11]}$ originating from the $\mathrm{d}^{n}$ (or $\mathrm{f}^{n}$ ) configurations of transitionmetal ions in the presence of coordinating ligands through a combination of the configuration interaction $(\mathrm{Cl})$ and KS-DFT approaches. In doing so, both dynamic correlation (by the DFT exchange-correlation potential) and nondynamic correlation (by $\mathrm{Cl}$ ) are considered. This procedure yields multiplet energies and fine-structure splitting. ${ }^{[10 b, c]}$ It has been shown that all common molecular properties, for example, zero-field splitting ${ }^{\left[10 g,{ }^{12]}\right.}$ Zeeman interactions, ${ }^{[13]}$ hyperfine splitting ${ }^{[13]}$ JahnTeller (JT) effects, ${ }^{[14]}$ magnetic exchange coupling ${ }^{[15]}$ shielding constants, $^{[16]}$ electronic structure and transitions in $f$ elements, ${ }^{[12 a, 17]}$ and $\mathrm{f}-\mathrm{d}$ transitions, ${ }^{[8 \mathrm{a}, 18]}$ can be calculated by this procedure. LF-DFT proved to be particularly good for determining the MAEs in trigonal-bipyramidal $\mathrm{Ni}^{2+}$ complexes ${ }^{[12 c]}$ and complexes of $\mathrm{Fe}^{+} .{ }^{[10]}$ In both cases, LF-DFT gave excellent agreement with highly accurate $\mathrm{MRCl}^{[12 \mathrm{c}]}$ and CASPT2 ${ }^{[10]}$ calculations, respectively. Furthermore, LF-DFT was able to calculate the very low experimentally observed value of MAE in the acetylacetonate $\cdot \mathrm{Cr}^{3+}$ complex, and it was even appropriate for calculations of the MAEs in excited states. ${ }^{[19]}$ In addition, we will show and prove that this method allows the analysis of the influence of relevant excited states on the MAE. The LFDFT method can be applied to complexes with a large number of atoms and even with no symmetry without a significant impact on valuable computational time. More importantly, values of variables that are familiar to experimentalists and that can be directly compared with experimental data are obtainable by using LF-DFT.

To examine the influence of different metal ions and their electronic configurations on the MAE, it is essential to choose a specific type of ligand that can bind a variety of metals. A suitable candidate is the "scorpionate"-type ligand hydrotris(pyrazolyl)borate (hereafter denoted as $\mathrm{Tp}^{-}$). However, there are still only a few papers dealing with the magnetic properties of transition-metal complexes with this particular kind of ligand..$^{[7 a, 20]}$

In the present study, the LF-DFT method was applied to analyze the influence of different metal ions and their electronic configurations on the MAEs in a series of $\mathrm{Tp}^{-}$metal complexes, namely $[\mathrm{VTpCl}]^{+},[\mathrm{CrTpCl}]^{+},[\mathrm{MnTpCl}]^{+},[\mathrm{FeTpCl}],[\mathrm{CoTpCl}]$, and $[\mathrm{NiTpCl}]$, and their methyl-substituted analogues (Figure $1 a, b) .{ }^{[20]}$ Furthermore, the systems with degenerate ground

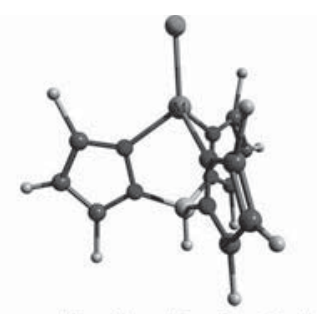

$\mathrm{M}=\mathrm{Ni}^{2+}, \mathrm{Co}^{2+}, \mathrm{Mn}^{3+}, \mathrm{Fe}^{2+}, \mathrm{Cr}^{3+}, \mathrm{V}^{3+}$
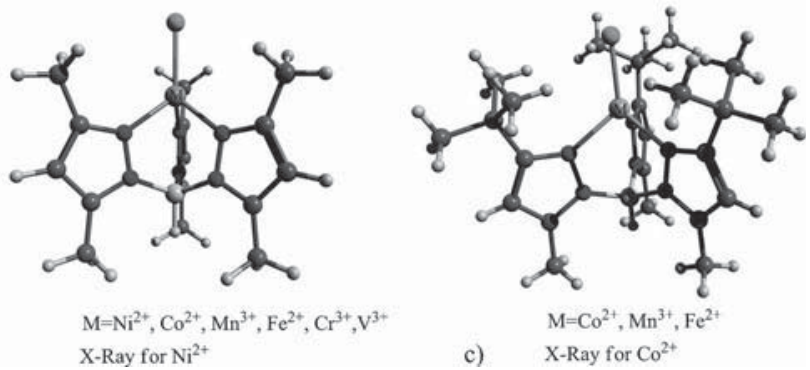

Figure 1. Structure of the investigated hydrotris(pyrazolyl)borate $\left(\mathrm{Tp}^{-}\right)$metal complexes: a) The unsubstituted systems, b) the methyl-substituted systems, and c) the tert-butyl-substituted systems.

states that undergo JT distortions, that is, $[\mathrm{MnTpCl}]^{+}$and $[\mathrm{FeTpCl}$, are of special interest due to the unquenched orbital moment, which leads, in many cases, to large MAE values. ${ }^{[21]}$ Because the JT effect competes with spin-orbit coupling, which in many cases leads to a reduction in the MAE in these systems after the distortion, ${ }^{[22]}$ we also examined in detail how substitution by methyl and tert-butyl groups in the $[\mathrm{MnTpCl}]^{+}$ and $[\mathrm{FeTpCl}]$ complexes (Figure $1 \mathrm{~b}, \mathrm{c}$ ) influences the distortion and, consequently, the value of the MAE. The primary objective of this study was to find ways to fine-tune the magnetic properties of these systems from the viewpoint of Equation (1).

The paper is organized as follows. First, we describe the theory behind LF-DFT, and briefly review the MAE. We then present and discuss the results before reporting our main conclusions. Finally, we describe the details of the computational method employed. 


\section{Theoretical background}

In all the structures under study, the central metal ion is tetracoordinated by one chloride ligand and three nitrogen atoms of $\mathrm{Tp}^{-}$. The specific structure of the $\mathrm{Tp}^{-}$ligand is responsible for the pseudotetrahedral $C_{3 v}$ geometry of the investigated complexes. It is noteworthy that even the substituted complexes (Figure 1) have $C_{3 v}$ symmetry. As a result of this pseudotetrahedral symmetry, the splitting between the d levels of the complex is generally small and favors the high-spin configuration in all the tested complexes. A schematic representation of the splitting of the $d$ orbitals for different configurations is given in Figure 2.

It should be pointed out that in $C_{3 v}$ symmetry, mixing between $e_{1(x z, y z)}$ and $e_{1\left(x y, x^{2}-y^{2}\right)}$ orbitals is allowed and does occur, and the labels in Figure 2 only indicate the main character of the orbital. This fact is clearly seen when representing the orbitals with predominantly $d$ character obtained from KS-DFT calculations (Figure 3).

The upper set of $e_{1}$ orbitals has a larger contribution of $d_{x z}$ and $d_{y z}$ orbitals, whereas in the lower set, the $d_{x y}$ and $d_{x^{2}-y^{2}}$ orbitals dominate. The interactions between $d$-metal orbitals and the $p$ orbitals of chlorine in the upper $e_{1}$ set are $\pi$ antibonding, whereas the lower $e_{1}$ orbitals are nonbonding with respect to the chloride ligand. The mixing of orbitals with $E$ symmetry gives rise to very interesting changes in the magnetic behavior

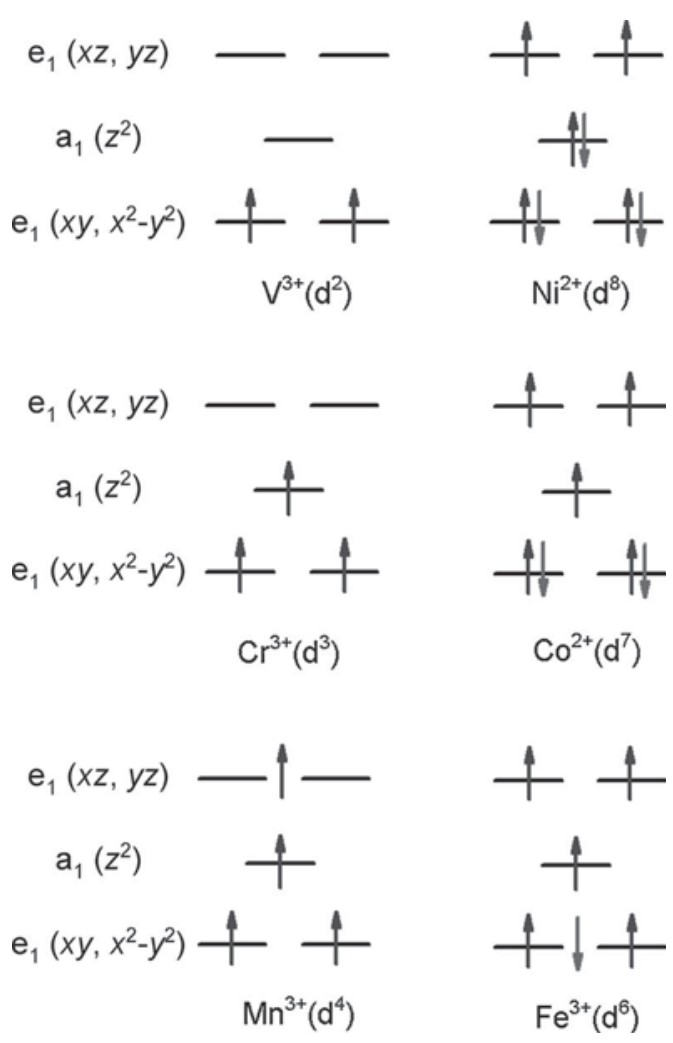

Figure 2. Splitting of the $d$ orbitals in the $C_{3 v}$ symmetry group and the distribution of electrons for different electronic configurations. Note that the $\mathrm{d}^{2} /$ $\mathrm{d}^{8}, \mathrm{~d}^{3} / \mathrm{d}^{7}$, and $\mathrm{d}^{4} / \mathrm{d}^{6}$ configurations of the $\mathrm{V}^{3+} / \mathrm{Ni}^{2+}, \mathrm{Cr}^{3+} / \mathrm{Co}^{2+}$, and $\mathrm{Mn}^{3+}$ / $\mathrm{Fe}^{2+}$ complexes, respectively, give rise to the same multiplets.

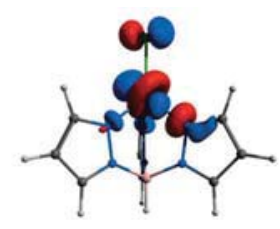

e1
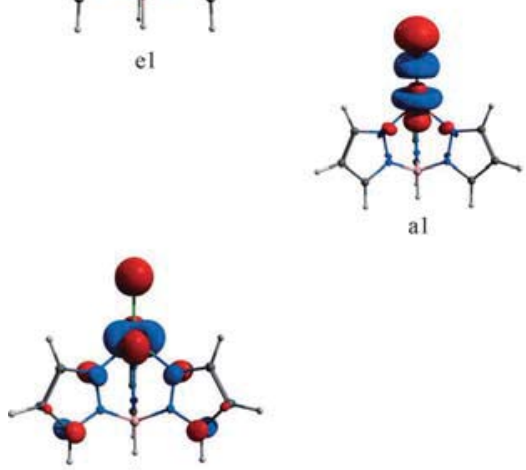

el

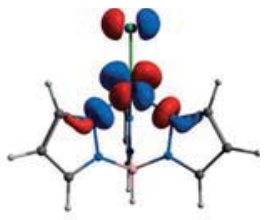

el

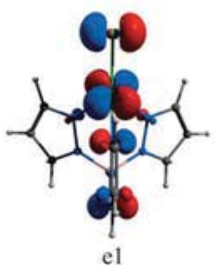

Figure 3. Molecular orbitals with predominantly d character, obtained from KS-DFT calculations.

of the investigated complexes, especially in structures with a degenerate ground state, as will be shown later.

Because the active electrons in the systems under study are localized over the metallic ion and its surrounding ligands, the Hamiltonian can be written as Equation (2), in which $\hat{H}_{\mathrm{ER}}, \hat{H}_{\mathrm{LF}}$ and $\hat{H}_{\text {so }}$ represent the electrostatic repulsion between the active electrons, ligand field, and spin-orbit interactions of the $3 \mathrm{~d}$ electrons of the metal ion, respectively.

$\hat{H}=\hat{H}_{\mathrm{ER}}+\hat{H}_{\mathrm{LF}}+\hat{H}_{\mathrm{SO}}$

$\hat{H}_{\mathrm{ER}}$ can be approximated in terms of the Racah parameters $B$ and $C$, which parametrize the radial part of its matrix elements, $\hat{H}_{\mathrm{LF}}$ represents both the electrostatic and bonding effects of the ligands on the metal, and is commonly expressed in terms of spherical harmonics functions $Y_{k, q}$ [see Eq. (S1) in the Supporting Information], and $\hat{H}_{\mathrm{so}}$ is the effective one-electron spin-orbit coupling (SOC) operator, which is expressed by Equation (3), in which the symbol $\alpha$ represents the fine-structure constant, $Z_{\text {eff }}^{(\chi)}$ the effective charge of the nucleus, $\xi$ the spin-orbit constant of the metal ion, and $r_{i \lambda}$ the distance between the electron and the nucleus.

$\hat{H}_{\mathrm{sO}}=\frac{1}{2} \alpha^{2} \sum_{i \lambda} \frac{Z_{\mathrm{eff}}^{(\mathrm{x})}}{r_{i \lambda}^{3}} \overrightarrow{\vec{S}}_{i \lambda} \vec{s}_{i}=\sum_{i} \vec{\zeta}\left(\vec{r}_{i}\right) \bar{s}_{i}$

In orbitally nondegenerate systems, the MAE of the ground state exists only due to the out-of-state SOC with the excited states and is measured by ZFS parameters. In particular, the ground-state splitting term is described by the spin Hamiltonian given in Equation (4), in which $D$ and $E$ represent the axial and rhombic ZFS parameters, respectively.

$\hat{H}=\left[D\left(S_{z}^{2}-\frac{S(S+1)}{3}+E\left(S_{x}^{2}-S_{\gamma}^{2}\right)\right]\right.$ 
By using DFT calculations combined with a ligand-field scheme, ${ }^{[10 \mathrm{~b}, \mathrm{c}]}$ the values of $D$ and $E$ can be derived from a comparison of the splittings in the lowest multiplet with the spin Hamiltonian given by Equation (5). ${ }^{[23]}$ It is noteworthy that in pure axial systems, for example, $C_{3 v}$ symmetry, as in the present cases, the $E$ parameter is equal to zero. In distorted structures of complexes with $S=2$, in addition to the well-defined ZFS parameters $D$ and $E$, additional fourth-order splitting parameters, $B_{4}, B_{4}{ }^{2}$, and $B_{4}{ }^{4}$, are needed to fully describe the fine-splitting of the ground-state term [see Eq. (S2) in the Supporting Information]. ${ }^{[23 b]}$ However, when the lowest term is orbitally degenerate, the MAE exists mostly due to the in-state SOC. The angular momentum is unquenched and Equation (4) is no longer valid. ${ }^{[2]}$ Thus, it is necessary to develop new Hamiltonians ${ }^{[12 c]}$ to describe the situation that exists, for example, in complexes involving $\mathrm{Mn}^{3+}$ and $\mathrm{Fe}^{2+}$ ions (see Figure 2). In both systems, the ground-state term is ${ }^{5} \mathrm{E}$ and it experiences first-order splitting by spin-orbit coupling. ${ }^{[21,25]}$ Thus, the nature of this splitting will be studied first by evaluating the spin-orbit Hamiltonian for the 10 microstates of the ${ }^{5} \mathrm{E}$ term.

To perform this calculation, it is important to remember that in cases in which a reflection plane exists perpendicular to the main axis of the molecule, then the $e_{\left(x^{2}-y^{2}, x y\right)}$ and $e_{(x z, y z)}$ orbitals do not mix. Each of these orbitals can then be expressed in terms of pure angular momentum functions, that is, $e_{\left(l_{z}=2, l_{2}=-2\right)}$ and $e_{\left(I_{2}=1, l_{2}=-1\right)}$ orbitals can be produced from $e_{\left(x^{2}-y^{2}, x y\right)}$ and $e_{(x z, y z)}$ orbitals, respectively. The splitting of the 10 microstates due to only the in-state SOC, as well as to the combined in-state and out-of state SOC, is presented in Figure 4 for the general cases $I=2$ and $I=1$.

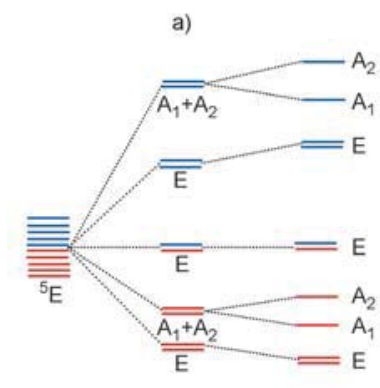

No SOC in-state SOC in-state

out-of state SOC

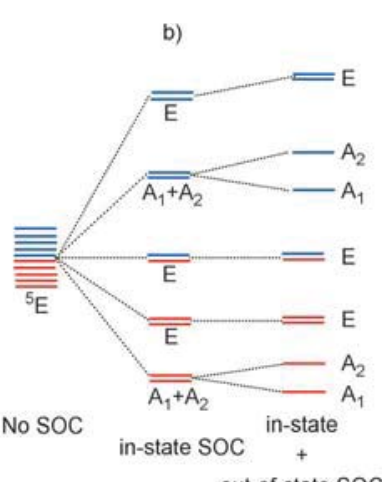

out-of state SOC
Figure 4. Splitting of the 10 microstates due to in-state SOC only and combined in-state and out-of state SOC for a) the $\mathrm{Fe}^{2+}$ complex and b) the $\mathrm{Mn}^{3+}$ complex.

On the other hand, when the symmetry plane does not exist, as in the complexes considered in the present study, $e_{\left(x^{2}-y^{2}, x y\right)}$ and $e_{(x z, y z)}$ mix and the resulting orbitals can be written as given by Equation (5).

$e_{ \pm}=\cos \theta e\left(I_{z}= \pm 2\right)+\sin \theta e\left(I_{z}=\mp 1\right)$

By using the orbitals in Equation (5) and the pure $S=2$ functions for the spin and orbital angular momentum terms, the
Hamiltonian matrix can be formed by evaluating the spinorbit operator. A similar reasoning was followed in ref. [21b]. The diagonal matrix given by Equation (6) is obtained.

$\left\langle{ }_{S_{z}}^{5} E_{\gamma}\left|H_{\mathrm{SOC}}\right|{ }_{S_{z}^{\prime}}^{5} E_{\gamma^{\prime}}\right\rangle=\xi \delta_{\lambda \gamma^{\prime}} \delta_{S_{z} S_{z}^{\prime}}\left(2 \cos ^{2} \theta+\sin ^{2} \theta\right) \frac{S_{z}}{4}$

In the above expression, $\gamma$ and $\gamma^{\prime}$ take \pm values like the orbitals in Equation (5). Because the $e_{ \pm}$orbitals do not interact through the spin-orbit Hamiltonian, it is clear that all the eigenvalues of Equation (6) are repeated twice (i.e., they are either degenerate or accidentally degenerate). According to Equation (6) (see also Figure 5), the spin-orbit levels of the

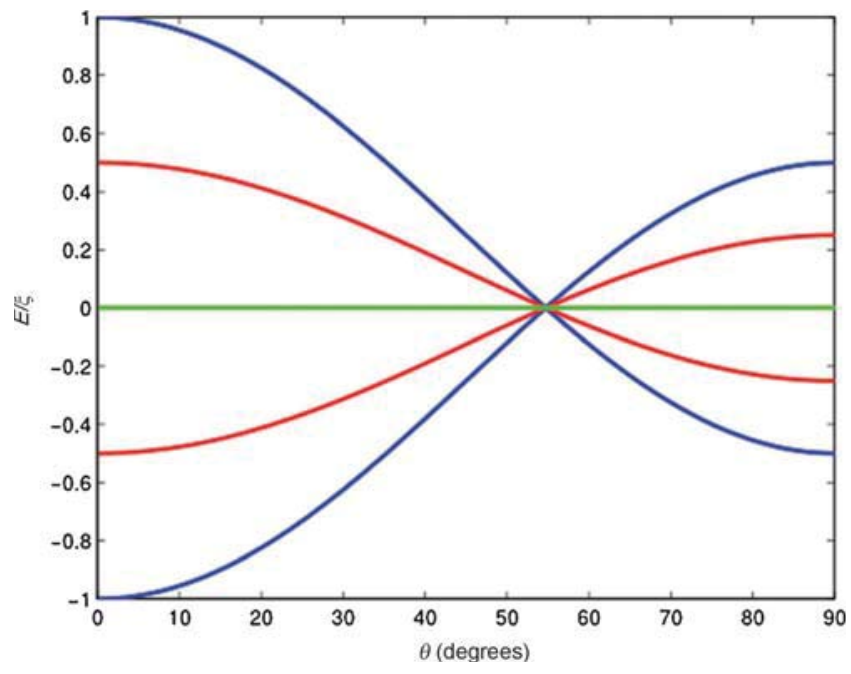

Figure 5. Diagram of the splitting of the ${ }^{5} \mathrm{E}$ term as the mixing of the $e_{\left(x^{2}-y^{2}, x y\right)} / e_{(x z, y z)}$ orbital changes as controlled by the angle $\theta$ described in Equation (6).

states in which pure $e_{\left(x^{2}-y^{2}, x y\right)}$ or $e_{(x z, y z)}$ orbitals are occupied would be equally separated by the quantity $U_{\text {in-state }}$ (the splitting due to the in-state SOC) with a total separation between the lowest and highest levels twice that in the case of $e_{\left(x^{2}-y^{2}, x y\right)}$ $[\theta=0$ or equivalent $]$ as compared with $e_{(x z, y z)}[\theta=\pi / 2$ or equivalent]. In intermediate cases, for which $0<\theta<\pi / 2$, the energies show a smooth transition between the cases with $I_{z}=2$ and $I_{z}=1$ with a highly degenerate crossing point for the "magic" angle $\tan ^{2} \theta=2 \leftrightarrow \theta \approx 54.7^{\circ}$, for which the magnetic anisotropy is zero when only the ground ${ }^{5} \mathrm{E}$ term is considered. This point corresponds to the point at which the wavefunctions behave approximately with a pure tetrahedral symmetry (see ref. [21b]) and the system becomes quasi-isotropic with a very small magnetic anisotropy. This proves that covalency with ligands controlling the mixing angle $\theta$ is determinant in the understanding of the magnetic properties of these systems. Thus, it is to be expected that these properties will largely be altered when the coordination of the transition metal is changed. Finally, it should be noted that the inclusion of out-of-state coupling with excited terms breaks the accidental degeneracy of two of the doublets transforming them into four singlets, 
yielding six excitations from the ground state for the system. Another important change occurring in the ordering of the spin-orbit levels can be observed when the orbital giving rise to the ground state is predominantly $I_{z}= \pm 1$ or $I_{z}= \pm 2$ (Figure 4). In particular, the lowest two states can be $A_{1}+A_{2}$, the separation of which is the magnetic tunneling splitting or $E$, in which case the magnetic tunneling is exactly zero. This means that the stability of a spin prepared for a complex in which the lowest state is $\mathrm{E}$ will be much larger (essentially zero in the absence of out-of-state SOC and external perturbation) than for an $A_{1}+A_{2}$ case. It should be noted that although this mechanism allows non-Kramers ions to have zero magnetic tunneling, similarly to Kramers ions, the degeneracy and spin stability in the latter are more resistant to perturbations because, for example, they cannot be altered by vibrations.

It is important to note that these Hamiltonian levels (five doubly degenerate states) are very different to those of an $S=2$ system (two doubly degenerate states and a singlet). Thus, this shows that Equation (4) cannot be used to describe the levels in this system.

Finally, we would like to note that the introduction of the JT effect, as necessary when a degenerate term is present, leads to a progressive quenching of the angular momentum and so to a smooth transition between the levels described by Equation (6) at high symmetry towards the levels associated with the Hamiltonian in Equation (4). ${ }^{[21 a, 25]}$

\section{Results and Discussion}

\section{Magnetic anisotropy in complexes with a nondegenerate ground state}

Selected structural parameters of the energy-minimized structures of the $[\mathrm{VTpCl}]^{+},[\mathrm{CrTpCl}]^{+},[\mathrm{CoTpCl}]$, and $[\mathrm{NiTpCl}]$ unsubstituted complexes and their methyl-substituted analogues with $C_{3 v}$ symmetry are presented in Table S1 in the Supporting Information. Comparison with the crystallographic data for $\left[\mathrm{NiTp}^{\mathrm{CH}}{ }_{3} \mathrm{Cl}\right]^{[20 \mathrm{~b}]}$ (see Table S1) indicates that all the bond lengths and angles are reproduced with a good degree of accuracy (the errors are close to $1 \%$ ). The $\mathrm{Ni}-\mathrm{Cl}$ and $\mathrm{Ni}-\mathrm{N}$ bond lengths in the $C_{3 v}$ optimized structure of $\left[\mathrm{NiTp}^{\mathrm{CH}}{ }_{3} \mathrm{Cl}\right]$ are 2.188 and $1.987 \AA$, respectively, whereas in the crystal structure they are 2.195 and $1.961 \AA$. In the optimized structure all three $\mathrm{Cl}-\mathrm{Ni}-\mathrm{N}$ angles are the same, $124,1^{\circ}$, whereas in the crystal structure, one is $122.4^{\circ}$ and the two others are $123.7^{\circ}$, thus breaking with the idealized $C_{3 v}$ symmetry. The differences between the geometrical parameters of the optimized and crystal structures are acceptable because the crystal structure is distorted as a result of the packing. Furthermore, the geometries obtained by DFT calculations are in good agreement with the previous results of Ye and Neese. ${ }^{[7 a]}$ These small differences in the geometries do not influence the magnetic properties in any significant way. The calculated excitation energies and the $D$ values for the unsubstituted and methyl-substituted complexes, obtained at the LF-DFT/OPBE level of theory, are tabulated in Table 1. All nonempirical parameters used in LF-DFT are summarized in Table S2.

Table 1. The excitation energies (in the absence of SOC) of the most important LFT transitions and the ZFS parameter $D_{\text {calcd. }}$

Complex Transition Transition Transition Transition Transition $D_{\text {calcd }}$ $\begin{array}{lllllll}1 & 2 & 3 & 4 & 5 & {\left[\mathrm{~cm}^{-1}\right]}\end{array}$

${ }^{3} \mathrm{~A}_{2} \rightarrow{ }^{3} \mathrm{E} \quad{ }^{3} \mathrm{~A}_{2} \rightarrow{ }^{3} \mathrm{~A}_{1} \quad{ }^{3} \mathrm{~A}_{2} \rightarrow{ }^{3} \mathrm{E} \quad{ }^{3} \mathrm{~A}_{2} \rightarrow{ }^{1} \mathrm{E} \quad{ }^{3} \mathrm{~A}_{2} \rightarrow{ }^{1} \mathrm{~A}_{1} \quad+2.00$

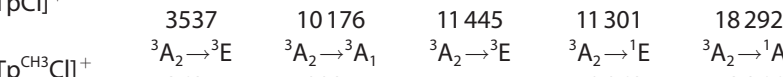

${ }^{3} \mathrm{~A}_{2} \rightarrow{ }^{3} \mathrm{E} \quad{ }^{3} \mathrm{~A}_{2} \rightarrow{ }^{3} \mathrm{~A}_{1} \quad{ }^{3} \mathrm{~A}_{2} \rightarrow{ }^{3} \mathrm{E} \quad{ }^{3} \mathrm{~A}_{2} \rightarrow{ }^{1} \mathrm{E} \quad{ }^{3} \mathrm{~A}_{2} \rightarrow{ }^{1} \mathrm{~A}_{1}$

$3624 \quad 9924 \quad 11144 \quad 10962 \quad 18866$

${ }^{4} \mathrm{~A}_{2} \rightarrow{ }^{4} \mathrm{E} \quad{ }^{4} \mathrm{~A}_{2} \rightarrow{ }^{4} \mathrm{~A}_{1} \quad{ }^{4} \mathrm{~A}_{2} \rightarrow{ }^{2} \mathrm{E} \quad{ }^{4} \mathrm{~A}_{2} \rightarrow{ }^{2} \mathrm{~A}_{1} \quad{ }^{4} \mathrm{~A}_{2} \rightarrow{ }^{2} \mathrm{E}$

$\begin{array}{lllll}10966 & 11898 & 10891 & 16170 & 16873\end{array}$

${ }^{4} \mathrm{~A}_{2} \rightarrow{ }^{4} \mathrm{E} \quad{ }^{4} \mathrm{~A}_{2} \rightarrow{ }^{4} \mathrm{~A}_{1} \quad{ }^{4} \mathrm{~A}_{2} \rightarrow{ }^{2} \mathrm{E} \quad{ }^{4} \mathrm{~A}_{2} \rightarrow{ }^{2} \mathrm{~A}_{1} \quad{ }^{4} \mathrm{~A}_{2} \rightarrow{ }^{2} \mathrm{E}$

$\begin{array}{lllll}10448 & 11008 & 11192 & 11222 & 16157\end{array}$

${ }^{4} \mathrm{~A}_{2} \rightarrow{ }^{4} \mathrm{E} \quad{ }^{4} \mathrm{~A}_{2} \rightarrow{ }^{4} \mathrm{~A}_{1} \quad{ }^{4} \mathrm{~A}_{2} \rightarrow{ }^{2} \mathrm{E} \quad{ }^{4} \mathrm{~A}_{2} \rightarrow{ }^{4} \mathrm{E} \quad{ }^{4} \mathrm{~A}_{2} \rightarrow{ }^{4} \mathrm{E}$

$\begin{array}{lllll}2240 & 6731 & 7734 & 7857 & 16579\end{array}$

${ }^{4} \mathrm{~A}_{2} \rightarrow{ }^{4} \mathrm{E} \quad{ }^{4} \mathrm{~A}_{2} \rightarrow{ }^{4} \mathrm{~A}_{1} \quad{ }^{4} \mathrm{~A}_{2} \rightarrow{ }^{2} \mathrm{E} \quad{ }^{4} \mathrm{~A}_{2} \rightarrow{ }^{4} \mathrm{E} \quad{ }^{4} \mathrm{~A}_{2} \rightarrow{ }^{4} \mathrm{E}$

$\begin{array}{lllll}2225 & 6909 & 7396 & 7901 & 16090\end{array}$

${ }^{3} \mathrm{~A}_{2} \rightarrow{ }^{3} \mathrm{E} \quad{ }^{3} \mathrm{~A}_{2} \rightarrow{ }^{3} \mathrm{~A}_{1} \quad{ }^{3} \mathrm{~A}_{2} \rightarrow{ }^{3} \mathrm{E} \quad{ }^{3} \mathrm{~A}_{2} \rightarrow{ }^{1} \mathrm{E} \quad{ }^{3} \mathrm{~A}_{2} \rightarrow{ }^{1} \mathrm{~A}_{1}$

$\begin{array}{lllll}7572 & 8697 & 12686 & 16580 & 17996\end{array}$

${ }^{3} \mathrm{~A}_{2} \rightarrow{ }^{3} \mathrm{E} \quad{ }^{3} \mathrm{~A}_{2} \rightarrow{ }^{3} \mathrm{~A}_{1} \quad{ }^{3} \mathrm{~A}_{2} \rightarrow{ }^{3} \mathrm{E} \quad{ }^{3} \mathrm{~A}_{2} \rightarrow{ }^{1} \mathrm{E} \quad{ }^{3} \mathrm{~A}_{2} \rightarrow{ }^{1} \mathrm{~A}_{1}$

$\begin{array}{lllll}7407 & 8274 & 12055 & 16475 & 17361\end{array}$

$+2.10$

0.50

$+0.03$

$+6.50$

$+8.65$

$+5.30$

$+5.38^{[a]}$

Results from LF-DFT obtained by using experimental geometries. $[\mathrm{CrTpCl}]^{+}$are comparable to the $D$ values determined for structurally similar complexes of $\mathrm{V}^{3+}$ and $\mathrm{Cr}^{3+} \cdot{ }^{[26]}$ Due to the very small values of $D$ for the $[\mathrm{VTpCl}]^{+}$and $[\mathrm{CrTpCl}]^{+}$complexes, the reliability of our calculations were verified by means of CASSCF/NEVPT2, and values of +1.33 and $0.10 \mathrm{~cm}^{-1}$ were obtained, respectively. ${ }^{[27]}$ These results are not surprising because $\mathrm{V}^{3+}$ has a very small spin-orbit $\left(209 \mathrm{~cm}^{-1}\right)^{[28]}$ and the $\mathrm{Cr}^{3+}$ levels in the complexes are almost degenerate, which indicates that this ion is almost perfectly tetrahedral. Somewhat higher values of $D$ were calculated for the $[\mathrm{CoTpCl}]$ and $[\mathrm{NiTpCl}] \mathrm{com}$ plexes. Although substitution of the methyl groups led to changes in the geometrical parameters (see Table S1 in the Supporting Information), the MAE was not drastically affected. ${ }^{[7 a]}$ For $\mathrm{Ni}^{2+}$ complexes, the spin-allowed transitions correspond to the ${ }^{3} \mathrm{E},{ }^{3} \mathrm{~A}_{1}$, and ${ }^{3} \mathrm{~A}_{2}$ excited multiplets. The calculated LF-DFT transitions agree reasonably with the experimental values of $6284\left({ }^{3} \mathrm{~A}_{2} \rightarrow{ }^{3} \mathrm{E}\right), 8204\left({ }^{3} \mathrm{~A}_{2} \rightarrow{ }^{3} \mathrm{~A}_{1}\right)$, and $11206 \mathrm{~cm}^{-1}$ $\left({ }^{3} \mathrm{~A}_{2} \rightarrow{ }^{3} \mathrm{E}\right)$. The value of $D$ calculated for the $\left[\mathrm{NiTp}^{\mathrm{CH}}{ }_{3} \mathrm{Cl}\right]$ crystal structure was in reasonable agreement with the experimentally obtained $D$ value of $3.9 \mathrm{~cm}^{-1}$. ${ }^{[20 \mathrm{~b}]}$ The distortion from the ideal $\mathrm{C}_{3 v}$ geometry in the crystal structure of $\left[\mathrm{NiTp}^{\mathrm{CH}}{ }_{3} \mathrm{Cl}\right]$ causes the existence of a rhombic ZFS parameter $E$. The value of $E$ calculated for the $\left[\mathrm{NiTp}_{3}{ }_{3} \mathrm{Cl}\right]$ crystal structure is $0.4 \mathrm{~cm}^{-1}$, whereas the experimentally obtained value is $0.35 \mathrm{~cm}^{-1}$. ${ }^{[20 \mathrm{~b}]}$ In the cases of the $\mathrm{Co}^{2+}$ complexes, the calculated transitions of nearly 7000 and $16000 \mathrm{~cm}^{-1}$ correspond well with the experimental bands at around 6000 and $16000 \mathrm{~cm}^{-1}$.20a] Furthermore, the calculated $D$ values are in accordance with the experimental values for the $\mathrm{Co}^{2+}$ scorpionate complexes (10.88-12.72). 
To understand the MAEs in the investigated complexes, the contributions of the most important transitions were analyzed. Because substitution does not lead to drastic changes in the $M A E$, in agreement with the arguments of Ye and Neese, ${ }^{[7 a]}$ the subsequent analysis will be focused mainly on the unsubstituted complexes (see Tables S3 and S4 for the $\mathrm{V}^{3+} / \mathrm{Ni}^{2+}$ and $\mathrm{Cr}^{3+} / \mathrm{Co}^{2+}$ complexes, respectively). Because the $\mathrm{d}^{2} / \mathrm{d}^{8}$ and $\mathrm{d}^{3} /$ $\mathrm{d}^{7}$ configurations of the $\mathrm{V}^{3+} / \mathrm{Ni}^{2+}$ and $\mathrm{Cr}^{3+} / \mathrm{Co}^{2+}$ complexes give rise to the same multiplets, this analysis could be realized by appropriate grouping of these complexes.

First, consider the contributions of the excited states of the $[\mathrm{VTpCl}]^{+}$and $[\mathrm{NiTpCl}]$ complexes to the MAE (see Table S3 in the Supporting Information). Because the ground states of both systems are nondegenerate ${ }^{3} A_{2}$ multiplets, ZFS exists due to out-of-state $S O C$ with the ${ }^{3} \mathrm{E}$ and ${ }^{3} \mathrm{~A}_{1}$ excited states (see Table S3). As shown below, non-negligible contributions also arise from ${ }^{1} \mathrm{E}$ and ${ }^{1} \mathrm{~A}_{1}$ spin-forbidden transitions.

The importance of the ${ }^{3} E$ and ${ }^{3} A_{1}$ excited states to the MAE were elucidated by exclusion of these states from the full Hamiltonian (Figure 6). Because all the systems behave qualitatively in a similar fashion, only the results for $[\mathrm{NiTpCl}]$ will be presented.

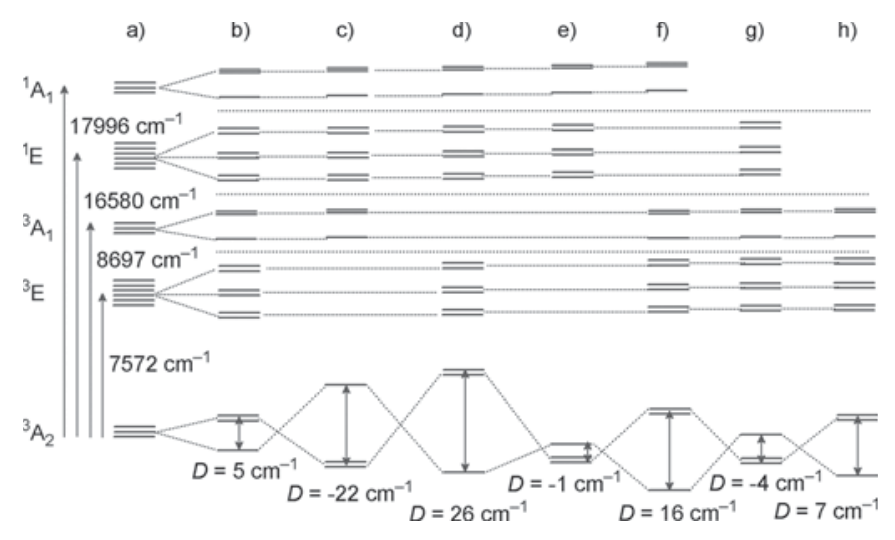

Figure 6. Diagram showing the effects of the low-energy triplet and singlet states on the MAE at the LF-DFT level for [NiTpCl]. a) Hamiltonian with only ligand field and electrostatic terms (MAE not defined). b) Full spin-orbit Hamiltonian evaluated with all states of the $d^{8}$ configuration. Results obtained with the full Hamiltonian when c) the ${ }^{3} E$ state is excluded, d) the ${ }^{3} A$ state is excluded, e) the ${ }^{3} \mathrm{E}$ and ${ }^{3} \mathrm{~A}_{1}$ states are excluded, $\mathrm{f}$ ) when the ${ }^{1} \mathrm{E}$ state is excluded, $g$ ) the ${ }^{1} \mathrm{~A}_{1}$ state is excluded, and $\mathrm{h}$ ) when both the ${ }^{1} \mathrm{E}$ and ${ }^{1} \mathrm{~A}_{1}$ states are excluded.

As indicated above, when all the states of a $d^{8}$ manifold were included in the diagonalization, the MAE was around $5 \mathrm{~cm}^{-1}$ (Figure $6 \mathrm{~b}$ ). The contribution of the first ${ }^{3} \mathrm{E}$ state to the total $D$ value is $27 \mathrm{~cm}^{-1}$ (see Table S3 in the Supporting Information). Hence, the exclusion of ${ }^{3} \mathrm{E}$ led to a negative $D$ value of about $-22 \mathrm{~cm}^{-1}$ (Figure $6 \mathrm{c}$ ). If the first ${ }^{3} A_{1}$ state, which gives a contribution of $-21 \mathrm{~cm}^{-1}$, was removed, the value of $D$ became even greater, but positive in sign $\left(26 \mathrm{~cm}^{-1}\right.$, Figure $\left.6 \mathrm{~d}\right)$. Finally, when both the ${ }^{3} \mathrm{E}$ and ${ }^{3} \mathrm{~A}_{1}$ states were excluded, the value of $D$ became negative again and equal to $-1 \mathrm{~cm}^{-1}$ (Figure 6e).

The removal of the first ${ }^{1} \mathrm{E}$ led to a high positive value of $D$ (Figure $6 f$ ), whereas the removal of ${ }^{1} A_{1}$ led to a negative value of $D\left(-4 \mathrm{~cm}^{-1}\right)$ (Figure $6 \mathrm{~g}$ ). The exclusion of both the ${ }^{1} \mathrm{E}$ and ${ }^{1} A_{1}$ states gave a positive $D$ value of $7 \mathrm{~cm}^{-1}$ (Figure $6 \mathrm{~h}$ ). These results corroborate the results of previously reported high-level ab initio calculations (CASSCF/NEVPT2/SORCI) ${ }^{[7 a]}$ that indicated significant contributions of the aforementioned transitions.

In the cases of the $\left[\mathrm{CrTpCl}^{+}\right.$and $[\mathrm{CoTpCl}]$ complexes (Kramers systems), the ground state is ${ }^{4} \mathrm{~A}_{2}$. The most important contributions to the $D$ value originate from interactions with the lowest ${ }^{4} \mathrm{E}$ and ${ }^{4} \mathrm{~A}_{1}$ excited states (see Table S4 in the Supporting Information). To analyze and obtain a deeper understanding of the way in which different states influence the magnetic anisotropy, calculations were performed in which again important states for [CoTpCl] were excluded (Figure 7).

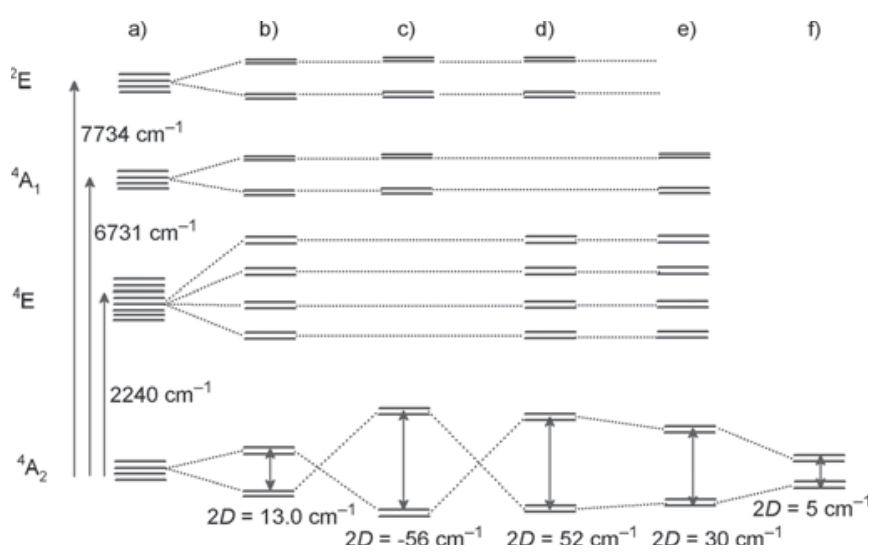

Figure 7. Diagram showing the effects of the low-energy states on the MAE at the LF-DFT level for [CoTpCl]. a) Hamiltonian with only ligand field and electrostatic terms (MAE not defined). b) Full spin-orbit Hamiltonian evaluated with all $d^{7}$ states. Results obtained with the full Hamiltonian when c) the ${ }^{4} E$ state, d) the ${ }^{4} A_{1}$ state, e) the ${ }^{2} E$ stat , and f) the three ${ }^{4} E,{ }^{4} A_{1}$, and ${ }^{2} E$ states were neglected in the calculation.

When all the states were included, the transition between states with $M_{s}= \pm 1 / 2$ and $M_{s}= \pm 3 / 2$ was $13.0 \mathrm{~cm}^{-1}$ (Figure $7 \mathrm{~b}$ ). When the first ${ }^{4} E$ state was excluded, the state with $M_{s}= \pm^{3} / 2$ became more stable and the transition was almost $56 \mathrm{~cm}^{-1}$ $\left(D=-28 \mathrm{~cm}^{-1}\right.$, Figure 7c). The removal of ${ }^{4} \mathrm{~A}_{1}$ or ${ }^{2} \mathrm{E}$ caused a stabilization of the state with $M_{s}= \pm 1 / 2$, that is, the value of $D$ became positive again (Figure 7d,e). Finally, when all three states, that is, ${ }^{4} E,{ }^{4} A_{1}$ and ${ }^{2} E$, were removed from the calculation, the MAE was drastically reduced (Figure $7 f$ ).

Summarizing the main results, for both the $[\mathrm{VTpCl}]^{+}$and [NiTpCl] complexes, the ${ }^{3} \mathrm{E}$ and ${ }^{1} \mathrm{~A}_{1}$ excited states contribute to the $D$ value with a positive sign, whereas the ${ }^{1} \mathrm{E}$ and ${ }^{3} \mathrm{~A}_{1}$ states contribute with a negative sign. However, the mutually opposing contributions largely cancel each other, leading to relatively small values of $D$ for both $[\mathrm{VTpCl}]^{+}$and $[\mathrm{NiTpCl}]$. The small differences in these contributions may be associated with the different transition energies of these states. Because these transitions are essentially one-electron excitations from the ground state, their energies could be altered by changing the ligand field, that is, it should be possible to increase the MAE by selecting stronger axial ligands. The higher contributions for $[\mathrm{NiTpCl}]$ than for $[\mathrm{VTpCl}]^{+}$are due to the larger SOC in $\mathrm{Ni}^{2+}$. 
The results can be easily explained by using simple group theory. The ground electronic state in both $[\mathrm{VTpCl}]^{+}$and [NiTpCl] is ${ }^{3} \mathrm{~A}_{2}$. In $\mathrm{C}_{3 v}$ symmetry, the orbital angular momentum operators $H_{\mathrm{SO}}{ }^{x, y}$ transform as $\mathrm{E}$, and $H_{\mathrm{sO}}{ }^{z}$ as $\mathrm{A}_{2}$, respectively. This implies that the only possible excitation via the $H_{\mathrm{sO}}{ }^{2}$ operator is from the ${ }^{3} \mathrm{~A}_{2}$ ground state to the ${ }^{3} \mathrm{~A}_{1}$ or ${ }^{1} \mathrm{~A}_{1}$ excited state $\left(A_{2} \otimes A_{2} \otimes A_{1}=A_{1}\right)$. Because coupling through the $H_{\text {so }}{ }^{2}$ operator is possible only if $\Delta M_{\mathrm{s}}=0$, the microstate with the value $M_{\mathrm{s}}=$ 1 , originating from the ${ }^{3} A_{2}$ ground state, can interact only with the $M_{\mathrm{s}}=1$ component of the ${ }^{3} \mathrm{~A}_{1}$ excited state (the same holds for $\left.M_{s}=-1\right)$. Consequently, the microstate of the ${ }^{3} \mathrm{~A}_{2}$ ground state with $M_{s}=0$ can interact only with the ${ }^{3} \mathrm{~A}_{1}$ excited state with $M_{s}=0$. The coupling is larger for larger $\left|S_{z}\right|$ values; thus, the ${ }^{3} A_{1}$ excited state contributes with a negative sign to the total value of $D$. The interaction with the ${ }^{1} A_{1}$ excited state through $H_{\mathrm{sO}}{ }^{2}$ is possible only between the $M_{\mathrm{s}}=0$ microstate; hence, the microstate with $M_{\mathrm{s}}=0$ is stabilized with respect to the $M_{\mathrm{s}}=1$ microstate. According to this, a positive contribution to the total $D$ value is obtained from the ${ }^{1} A_{1}$ excited state. Coupling through $H_{\mathrm{SO}}{ }^{x, y}$ is possible only between the ${ }^{3} \mathrm{~A}_{2}$ ground state and the ${ }^{3} E$ and ${ }^{1} E$ excited states $\left(A_{2} \otimes E \otimes E=A_{1}+\right.$ $\left.A_{2}+E\right)$. Furthermore, the interaction between these states is possible only if $\Delta M_{s}= \pm 1$. Thus, the microstate $M_{s}=1$ of ${ }^{3} \mathrm{~A}_{2}$ can interact only with $M_{\mathrm{s}}=0$ of ${ }^{3} \mathrm{E}$ (the same holds for $M_{\mathrm{s}}=-1$ of ${ }^{3} \mathrm{~A}_{2}$ ). However, the microstate $M_{s}=0$ of ${ }^{3} \mathrm{~A}_{2}$ can interact with both the $M_{s}=-1$ and $M_{s}=1$ microstates of ${ }^{3} \mathrm{E}$. As a consequence, the stabilization of $M_{s}=0$ is larger, and hence there is a positive contribution to the total $D$ value from ${ }^{3} \mathrm{E}$. When ${ }^{1} \mathrm{E}$ couples with the ${ }^{3} \mathrm{~A}_{2}$ ground state, only $M_{\mathrm{s}}=1$ (and $M_{\mathrm{s}}=-1$ ) of the ground state can interact with $M_{\mathrm{s}}=0$ of ${ }^{1} \mathrm{E}$, leading to negative contributions to $D$. A similar analysis can be made for the $[\mathrm{CrTpCl}]^{+}$and $[\mathrm{CoTpCl}]$ complexes. In the cases of the $[\mathrm{CrTpCl}]^{+}$and $[\mathrm{CoTpCl}]$ complexes, ${ }^{4} \mathrm{E}$ and ${ }^{4} \mathrm{~A}_{1}$ contribute with a positive and a negative sign, respectively.

\section{Magnetic anisotropy in complexes with a degenerate ground state}

The complexes with $d^{4}$ or $d^{6}$ high-spin configuration, that is, $[\mathrm{MnTpCl}]^{+}$and $[\mathrm{FeTpCl}]$, are in a ${ }^{5} \mathrm{E}$ ground state for the $C_{3 \mathrm{v}}$ point group. Systems with degenerate ground states are of special interest due to the unquenched orbital moment that leads to a large MAE. However, species with degenerate states are JT-active. In the cases of the $\left[\mathrm{MnTpCl}^{+}\right.$and $[\mathrm{FeTpCl}] \mathrm{com}$ plexes, and according to group theory considerations, the lowering of symmetry leads to a change from the $C_{3 v}$ to the $C_{s}$ point group. $\mathrm{Tp}^{-}$is a rigid chelate ligand, and the JT distortion is manifested through changes in the $\mathrm{Cl}-\mathrm{M}-\mathrm{N}$ angles, denoted as $\alpha$ and $\beta$ and illustrated in Figure 8. Although JT distortion leads to a reduction in the MAE, it was previously shown that it could be quenched by the careful choice of ligands. ${ }^{[12 c]} \mathrm{A}$ practical way to suppress $\mathrm{JT}$ distortion in the $[\mathrm{MnTpCl}]^{+}$and $[\mathrm{FeTpCl}]$ complexes could be the substitution of the hydrogen atoms on the pyrazolyl rings with voluminous groups. Thus, the effect of substitution of the hydrogen atoms on the pyrazolyl rings with methyl and tert-butyl groups on the JT distortion was analyzed in detail. The structure and shape of the

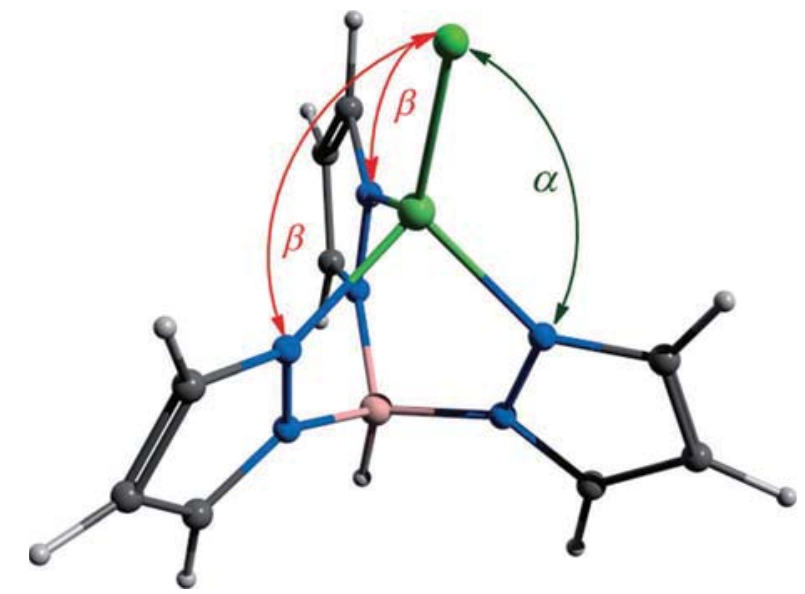

Figure 8. Structure of the investigated complexes with the angles $\alpha$ and $\beta$ depicted. JT distortion manifests itself mainly through changes in the $\alpha$ and $\beta$ angles.

substituents do not reduce the initial $C_{3 v}$ symmetry by themselves. Hence, the substituted $[\mathrm{MnTpCl}]^{+}$and $[\mathrm{FeTpCl}] \mathrm{com}-$ plexes also have $C_{3 v}$ symmetry. The lowering of symmetry due to JT distortion is from the $C_{3 v}$ to the $C_{s}$ point group, as in the cases of the unsubstituted complexes. The structural parameters and the JT stabilization energies of all the optimized structures are summarized in Table 2.

Table 2. Selected bond lengths and angles for the DFT-optimized structures of the investigated $\mathrm{Mn}^{3+}$ and $\mathrm{Fe}^{2+}$ complexes, as well as the JT stabilization energies.

\begin{tabular}{|c|c|c|c|c|c|c|c|}
\hline Complex & $\mathrm{CF}$ & $\begin{array}{l}R(\mathrm{M}-\mathrm{Cl}) \\
{[\AA]}\end{array}$ & $\begin{array}{l}R\left(\mathrm{M}-\mathrm{N}_{1}\right) \\
{[\AA]}\end{array}$ & $\begin{array}{l}R\left(\mathrm{M}-\mathrm{N}_{2}\right) \\
{[\AA]}\end{array}$ & $\begin{array}{l}\alpha \\
{\left[{ }^{\circ}\right]}\end{array}$ & $\begin{array}{l}\beta \\
{\left[{ }^{\circ}\right]}\end{array}$ & $\begin{array}{l}E_{\mathrm{JT}} \\
{\left[\mathrm{cm}^{-1}\right]}\end{array}$ \\
\hline \multirow{2}{*}[\mathrm{MnTpCl}]{$^{+}$} & $C_{3 v}$ & 2.152 & 1.994 & 1.994 & 124.3 & 124.3 & - \\
\hline & $C_{s}$ & 2.141 & 1.947 & 2.008 & 149.8 & 108.9 & 1457 \\
\hline \multirow{2}{*}[\mathrm{MnTp}{}^{\mathrm{CH}3}\mathrm{Cl}]{$^{+}$} & $C_{3 v}$ & 2.179 & 1.995 & 1.995 & 123.1 & 123.1 & - \\
\hline & $C_{s}$ & 2.171 & 1.928 & 2.011 & 139.1 & 114.5 & 960 \\
\hline \multirow{2}{*}[\mathrm{MnTp}{}^{\mathrm{tBu},\mathrm{CH}3}\mathrm{Cl}]{$^{+}$} & $C_{3 v}$ & 2.209 & 2.031 & 2.031 & 121.8 & 121.8 & - \\
\hline & $C_{s}$ & 2.205 & 2.146 & 1.993 & 132.1 & 116.1 & 830 \\
\hline \multirow{2}{*}{ [FeTpCl] } & $C_{3 v}$ & 2.197 & 2.072 & 2.072 & 124.6 & 124.6 & - \\
\hline & $C_{s}$ & 2.192 & 2.094 & 2.079 & 120.5 & 127.6 & 130 \\
\hline \multirow{2}{*}[\mathrm{FeTp}{}^{\mathrm{CH}3}\mathrm{Cl}]{} & $C_{3 v}$ & 2.216 & 2.062 & 2.062 & 124.0 & 124.0 & - \\
\hline & $C_{s}$ & 2.211 & 2.089 & 2.066 & 121.1 & 126.2 & 81 \\
\hline \multirow{2}{*}[\mathrm{FeTp}{}^{t\mathrm{Bu},\mathrm{CH}3}\mathrm{Cl}]{} & $C_{3 v}$ & 2.239 & 2.086 & 2.086 & 123.3 & 123.3 & - \\
\hline & $C_{s}$ & 2.232 & 2.076 & 2.115 & 127.7 & 120.5 & 46 \\
\hline
\end{tabular}

In all the $\mathrm{Mn}^{3+}$ complexes studied, shorter $\mathrm{M}-\mathrm{Cl}$ and $\mathrm{M}-\mathrm{N}$ bond lengths were obtained in comparison to the corresponding complexes of $\mathrm{Fe}^{2+}$, which is due to the higher charge of the $\mathrm{Mn}^{3+}$ ion. The difference in the $\alpha$ angle of the $C_{3 v}$ and $C_{s}$ conformations for $[\mathrm{MnTpCl}]^{+}$is $25.5^{\circ}$. On the other hand, the $\alpha$ angle for the $[\mathrm{FeTpCl}]$ minimum structure is $4.1^{\circ}$ smaller than in the HS structure. In addition, the JT stabilization energy for $[\mathrm{MnTpCl}]^{+}$is much larger than that for $[\mathrm{FeTpCl}]$. In the case of the $[\mathrm{MnTpCl}]^{+}$complex, the unpaired electron is placed in the upper $e$ orbital set. The interactions between the $d$-metal orbitals and the $\mathrm{p}$ orbitals of chlorine in the upper $e$ orbital set are $\pi$ antibonding (Figure 3 ). These antibonding interactions 
are drastically reduced in $C_{s}$ distorted structures. In other words, the displacement of the chlorine atom away from the $z$ axis will strongly affect the energy of the $d_{x z}$ and $d_{y z}$ orbitals of the system. On the other hand, in the $[\mathrm{FeTPCl}]$ complex, the unpaired electron is placed in the nonbonding lower $e$ orbital set. Hence the stabilization of the system through the deviation of the $\alpha$ angle is much smaller. The difference between the angles $\alpha$ of the $C_{3 v}$ and $C_{s}$ minimum conformations of the methyl-substituted $[\mathrm{MnTpCl}]^{+}$is $16^{\circ}$. This angle deviation is much lower than the angle deviation in the unsubstituted $[\mathrm{MnTpCl}]^{+}$(Table 2.). Accordingly, $E_{\mathrm{JT}}$ is reduced from 1457 to $960 \mathrm{~cm}^{-1}$. Furthermore, the tert-butyl-substituted analogue shows even smaller distortions, with a difference in $\alpha$ of only $10.3^{\circ}$. An even more noticeable effect was achieved by substitution of the hydrogen atoms in the $[\mathrm{FeTpCl}]$ complex, that is, the JT distortion became very weak with a stabilization energy of less than $100 \mathrm{~cm}^{-1}$ for both the methyl- and tert-butyl-substituted complexes.

The MAE was analyzed for both the $C_{3 v}$ and $C_{s}$ optimized structures of the investigated complexes. For the sake of a better understanding, first the MAE of the $\mathrm{Mn}^{3+}$ and $\mathrm{Fe}^{2+}$ complexes in $C_{3 v}$ symmetry will be analyzed and discussed (Table 3) and then an explanation will be given of how the JT distortion influences the MAEs of the systems.

The excitation energies from the lowest state of the ${ }^{5} \mathrm{E}$ term split by SOC are presented in the second column of Table 3. As indicated above, inclusion of the out-of-state coupling destroys the accidental degeneracies and splits the five doublets into three doublets and four singlets leading to six excitation energies. As can be observed in Table 3, the splits in the $\mathrm{Mn}^{3+}$ complexes are much smaller than in those containing $\mathrm{Fe}^{2+}$. Part of this effect can be explained by noting that the SOC constant is smaller for the $\mathrm{Mn}^{3+}$ ion than for the $\mathrm{Fe}^{2+}$ ion $\left(352 \mathrm{~cm}^{-1}\right.$ for the $\mathrm{Mn}^{3+}$ ion and $410 \mathrm{~cm}^{-1}$ for the $\mathrm{Fe}^{2+}$ ion), ${ }^{[28]}$ but it was observed that bonding plays a more important role. Using the orbital population of the active orbitals enables the angle $\theta$ to be estimated by measuring the amount of intermixing between the nonbonding $e_{\left(x^{2}-y^{2}, x y\right)}$ and the antibonding $e_{(x z, y z)}$ orbitals. As shown in Figure 3, a simple model predicts a very small anisotropy for mixings with a $\theta$ value of about $54.7^{\circ}$ as the system becomes quasitetrahedral, that is, more isotropic. Indeed, full calculations revealed that $\mathrm{Mn}^{3+}$ complexes have $\theta$ values in the vicinity of this magic angle and have very low values of $U_{\text {in-state. }}$ In particular, the value of $\theta$ for $[\mathrm{MnTpCl}]^{+}$is almost coincident with the magic angle and the splitting of the ${ }^{5} \mathrm{E}$ term is surprisingly dominated by the out-ofstate coupling with a very small $U_{\text {in-state }}$ value of $1.6 \mathrm{~cm}^{-1}$. Studying the other $\mathrm{Mn}^{3+}$ complexes, it was observed that the further their $\theta$ angles deviate from $54.7^{\circ}$, the larger the MAE becomes, as it is dominated by the linear $U_{\text {in-state }}$ On the other hand, the angles for the $\mathrm{Fe}^{2+}$ complexes deviate substantially from the critical degeneracy angle and thus their MAEs are larger than those of the $\mathrm{Mn}^{3+}$ systems. Still, the value of the MAE is directly related to the deviation of $\theta$ from $54.7^{\circ}$, increasing along the series $\left[\mathrm{FeTp}^{\mathrm{tBu}, \mathrm{CH} 3} \mathrm{Cl}\right]<\left[\mathrm{FeTp}{ }^{\mathrm{CH} 3} \mathrm{Cl}\right]<[\mathrm{FeTpCl}]$ as the angle decreases from 37.2 to $29.9^{\circ}$. This shows that covalency, as controlled by chemical substitution, is a key factor controlling the MAE in systems presenting orbital degeneracy.

As stated in the Theoretical Background section, the lowest states in the $\mathrm{Mn}^{3+}$ complexes are of type $\mathrm{A}_{1}+\mathrm{A}_{2}$, presenting finite magnetic tunneling. In fact, the magnetic splitting was observed to be quite large (greater than $1 \mathrm{~cm}^{-1}$ ), which would clearly prevent spin stability on macroscopic timescales. For tunnelings of less than $0.1 \mathrm{~cm}^{-1}$, it may be possible to apply an a.c. magnetic field that allows the observation of SMM behavior. ${ }^{[29]}$ On the other hand, $\mathrm{Fe}^{2+}$ complexes display $\mathrm{E}$ ground states and, as a consequence, show no tunneling splitting and thus their spin states should be considerably more stable than those of $\mathrm{Mn}^{3+}$ complexes.

The MAEs in low-symmetry $C_{s}$ structures are analyzed and explained here (Table 4). All the parameters are defined by the effective Hamiltonian approach, and were obtained by LF-DFT.

Table 3. Calculated excitation energies, $U$, for the $\mathrm{Mn}^{3+}$ and $\mathrm{Fe}^{2+}$ complexes with $\mathrm{C}_{3 \mathrm{v}}$ symmetry and the composition of the degenerate orbitals containing an unpaired electron. The compositions of the orbitals were extracted from AOC calculations.

\begin{tabular}{|c|c|c|c|c|}
\hline Complex & \multicolumn{2}{|c|}{$\begin{array}{c}U_{\text {(in-state }+ \text { out-of-state) }} \\
{\left[\mathrm{cm}^{-1}\right]}\end{array}$} & $\begin{array}{l}U_{\text {(in-state) }} \\
{\left[\mathrm{cm}^{-1}\right]}\end{array}$ & $\begin{array}{l}\text { Compositions of the orbitals } \\
\text { with unpaired electron }\end{array}$ \\
\hline$[\mathrm{MnTpCl}]^{+}$ & $\begin{array}{c}A_{1}, A_{2} \\
E \\
E \\
E \\
A_{1}, A_{2}\end{array}$ & $\begin{array}{c}0.0,12.0 \\
12.0 \\
23.0 \\
32.0 \\
35.0,42.0\end{array}$ & 1.6 & $\begin{array}{c}\mathrm{d}_{y z z^{\prime}} \mathrm{d}_{x z}(46.20 \%) \\
\mathrm{d}_{x y} \mathrm{~d}_{x^{2}-y^{2}}(22.30 \%) \\
\theta \approx 55.2^{\circ}\end{array}$ \\
\hline$\left[\mathrm{MnTp}{ }^{\mathrm{CH} 3} \mathrm{Cl}\right]^{+}$ & $\begin{array}{c}A_{1}, A_{2} \\
E \\
E \\
A_{1}, A_{2} \\
E\end{array}$ & $\begin{array}{c}0.0,3.0 \\
18.0 \\
34.0 \\
43.0,58.0 \\
61.0\end{array}$ & 12.2 & $\begin{array}{c}\mathrm{d}_{y z z^{\prime}} \mathrm{d}_{x z}(42.03 \%) \\
\mathrm{d}_{x y y} \mathrm{~d}_{x^{2}-y^{2}}(26.31 \%) \\
\theta \approx 51.6^{\circ}\end{array}$ \\
\hline$\left[\mathrm{MnTp}^{\mathrm{tBu}, \mathrm{CH} 3} \mathrm{Cl}\right]^{+}$ & $\begin{array}{c}A_{1}, A_{2} \\
E \\
E \\
A_{1}, A_{2} \\
E\end{array}$ & $\begin{array}{c}0.0,0.10 \\
32.0 \\
62.0 \\
85.0,100.0 \\
119.0\end{array}$ & 28.0 & $\begin{array}{c}\mathrm{d}_{y z z^{\prime}} \mathrm{d}_{x z}(37.49 \%) \\
\mathrm{d}_{x y} \mathrm{~d}_{x^{2}-y^{2}}(32.01 \%) \\
\theta \approx 47.3^{\circ}\end{array}$ \\
\hline [FeTpCl] & $\begin{array}{c}E \\
A_{1}, A_{2} \\
E \\
E \\
A_{1}, A_{2}\end{array}$ & $\begin{array}{c}0.0 \\
101.0,115.0 \\
214.0 \\
318.0 \\
422.0,422.0\end{array}$ & 107.0 & $\begin{array}{c}\mathrm{d}_{x y,} \mathrm{~d}_{x^{2}-y^{2}}(64.10 \%) \\
\mathrm{d}_{y z \prime}, \mathrm{d}_{x z}(21.20 \%) \\
\theta \approx 29.9^{\circ}\end{array}$ \\
\hline$\left[\mathrm{FeTp}{ }^{\mathrm{CH} 3} \mathrm{Cl}\right]$ & $\begin{array}{c}E \\
A_{1}, A_{2} \\
E \\
E \\
A_{1}, A_{2}\end{array}$ & $\begin{array}{c}0.0 \\
88.0,106.0 \\
192.0 \\
286.0 \\
380.0,381.0\end{array}$ & 97.0 & $\begin{array}{c}\mathrm{d}_{x y y} \mathrm{~d}_{x^{2}-y^{2}}(59.13 \%) \\
\mathrm{d}_{y z \prime} \mathrm{d}_{x z}(24.23 \%) \\
\theta \approx 32.6^{\circ}\end{array}$ \\
\hline$\left[\mathrm{FeTp}{ }^{\mathrm{tBu}, \mathrm{CH} 3} \mathrm{Cl}\right]$ & $\begin{array}{c}E \\
A_{1}, A_{2} \\
E \\
E \\
A_{1}, A_{2}\end{array}$ & $\begin{array}{c}0.0 \\
65.0,86.0 \\
155.0 \\
231.0 \\
308.0,309.0\end{array}$ & 78.0 & $\begin{array}{c}\mathrm{d}_{x y y} \mathrm{~d}_{x^{2}-y^{2}}(52.92 \%) \\
\mathrm{d}_{y z \prime}, \mathrm{d}_{x z}(30.40 \%) \\
\theta \approx 37.2^{\circ}\end{array}$ \\
\hline
\end{tabular}


Table 4. Calculated $D$ and $E$ values for the investigated complexes of $\mathrm{Mn}^{3+}$ and $\mathrm{Fe}^{2+}$ in $\mathrm{C}_{s}$ symmetry.

\begin{tabular}{lcl|} 
Complex & $D\left[\mathrm{~cm}^{-1}\right]$ & $E\left[\mathrm{~cm}^{-1}\right]$ \\
\hline$\left[\mathrm{MnTpCl}^{+}\right.$ & 4.66 & 0.50 \\
{$\left[\mathrm{MnTp}^{\mathrm{CH3}} \mathrm{Cl}\right]^{+}$} & 4.59 & 0.54 \\
{$\left[\mathrm{MnTp}^{\mathrm{BB}}, \mathrm{CH3} \mathrm{Cl}\right]^{+}$} & -4.50 & 0.04 \\
{$[\mathrm{FeTpCl}]$} & -22.92 & 1.54 \\
{$\left[\mathrm{FeTp}{ }^{\mathrm{CH3}} \mathrm{Cl}\right]$} & -22.62 & 2.03 \\
\hline
\end{tabular}

The calculated fourth-order parameters are listed in Table S5 in the Supporting Information.

The first transition in the minimum structure of [ $\mathrm{FeTpCl}$ is quenched to approximately $60 \%$ of the value of the high-symmetry conformation $\left(62 \mathrm{~cm}^{-1}\right.$ vs. $\left.101 \mathrm{~cm}^{-1}\right)$. However, this value still represents a large magnetic anisotropy in comparison with the minimum structure of $[\mathrm{MnTpCl}]^{+}$. Owing to the large distortion in $[\mathrm{MnTpCl}]^{+}$, the first transition is decreased in the $C_{s}$ structure and the $D$ value is positive. In addition, the value of $\xi$ for $[\mathrm{FeTPCl}]$ is nearly three times larger than $E_{\text {JT }}$ hence the SOC dominates over vibronic coupling. ${ }^{[12 c]}$ The differences between the MAEs in the $C_{3 v}$ and low-symmetry structures are less pronounced in comparison with the unsubstituted analogues due to the quenching of JT distortion upon substitution. The $D$ value for the $C_{s}$ structure of $\left[\mathrm{MnTp}^{\mathrm{CH} 3} \mathrm{Cl}^{+}\right.$is positive, a strong indication that the in-state SOC plays a very small role in these systems, as shown above. However, $\left[\mathrm{MnTp}^{\mathrm{tBu}, \mathrm{CH} 3} \mathrm{Cl}\right]^{+}$in the $C_{s}$ conformation exhibits a negative $D$ value. In the case of the $\left[\mathrm{FeTp}{ }^{\mathrm{CH}} \mathrm{Cl}\right]$ complex, the MAE is quenched compared with the value for the high-symmetry configurations. Moreover, in all the $\mathrm{Fe}^{2+}$ complexes studied, the distortion splits the lowest $\mathrm{E}$ state, thereby activating magnetic tunneling that facilitates spin decay even at $T=0 \mathrm{~K}$.

\section{Conclusion}

Even though SMMs have received considerable attention since their discovery, and it is well known that magnetic anisotropy is a crucial quantity, it is still difficult to control its value. Hence, it is of the utmost importance to understand all the different factors that could influence and enhance magnetic anisotropy.

We have reported herein a detailed analysis of scorpionate complexes with first-row transition-metal ions to probe the origin of magnetic anisotropy and to attempt to understand the chemistry and physics underlying it. For this purpose, LFDFT was employed as a method already proven to be accurate for these types of calculations and as a faster alternative to wavefunction-based methods.

Magnetic anisotropy arises as a consequence of two basic mechanisms: 1) In-state orbital angular momentum (degenerate ground states) and 2) out-of-state orbital angular momentum (spin-orbit mixing of certain excited states with the ground state). In the present study, both were treated.

For complexes with a nondegenerate ground state, it is necessary to be able to pin-point the particular excitations that control the sign and magnitude of the ZFS parameter, $D$. It was found in these systems that there are positive contributions to $D$ from the ${ }^{3} \mathrm{E}$ and ${ }^{1} \mathrm{~A}_{1}$ states in the cases of the $\mathrm{V}^{3+}$ and $\mathrm{Ni}^{2+}$ complexes, and due to the ${ }^{4} \mathrm{E}$ state in the cases of the $\mathrm{Cr}^{3+}$ and $\mathrm{Co}^{2+}$ complexes, whereas there are negative contributions to the $D$ value from the ${ }^{1} \mathrm{E}$ and ${ }^{3} \mathrm{~A}_{1}$ states for the $\mathrm{V}^{3+}$ and $\mathrm{Ni}^{2+}$ complexes, and from the ${ }^{4} \mathrm{~A}_{1}$ and ${ }^{2} \mathrm{E}$ states for the $\mathrm{Cr}^{3+}$ and $\mathrm{Co}^{2+}$ complexes. A delicate balance between these excited states yields the overall $D$ value, which in most cases is very small. It was found that these excitations are mainly controlled by the ligand field and so the MAE could be engineered by choosing suitable different first-neighbors to the metal. On the other hand, substitution of the hydrogen atoms on the pyrazolyl rings did not significantly affect the value of the MAE.

In the complexes with degenerate ground states, several competing effects influence the value of the magnetic anisotropy. Spin-orbit coupling gives the order of magnitude of the MAE as a result of the unquenched orbital angular momentum. In $C_{3 v}$ symmetry, the type of unequally populated $e$ orbitals and the degree of mixing between two sets of $e$ orbitals further determine the value of the MAE. In particular, it was shown that the MAE in these systems is controlled by this mixing, becoming negligible for a particular "magic" angle that makes the wavefunction behave like a quasi-isotropic tetrahedral one. In all the calculations, it was found that the MAE increases the further the system deviates from this particular angle, which controls the mixing of the orbitals. There are several ways to control this mixing angle, for example, the selection of particular ions that would predominantly fill either the anti- or nonbonding $e$ orbitals that are naturally at different distances from the "magic" angle. Moreover, the introduction of substituents onto the pyrazolyl rings also alters this angle, allowing chemical manipulation of the MAE. Moreover, selection of the correct ion ( $\mathrm{Fe}^{2+}$ in this case) may lead to systems that present zero magnetic tunneling for symmetry reasons, which should improve the stability of the spin states in nonKramers systems at very low temperatures. Finally, it should be emphasized that both the MAE and magnetic tunneling are very sensitive to the JT distortion occurring in these complexes. It was shown previously that changes of the substituents also allows the JT distortion to be quenched, which, in general, reduces the MAE of any given high-symmetry complex. Thus, this study has shown large differences between systems with and without orbital degenerate ground states and opens the way to chemically control the magnetic properties.

\section{Computational Methods}

Geometry optimizations calculations were performed by using a generalized gradient (GGA) functional consisting of OPTX ${ }^{[30]}$ for exchange and $\mathrm{PBEC}^{[31]}$ for correlation (OPBE). ${ }^{[32]}$ The multiplet structures of the investigated complexes were calculated by using the LF-DFT method. The LF-DFT procedure consists of the following steps: 1) An average-of-configuration (AOC) spin-restricted calculation with $n$ electrons distributed evenly over the five Kohn-Sham (KS) molecular orbitals dominated by the d orbitals of the metal, 
2) starting from the KS AOC orbitals, the energies of all Slater determinants (SDs) originating from the $\mathrm{d}^{n}$ shell were calculated in a spin-unrestricted way, 3 ) the SD energies and components of the corresponding KS AOC eigenvectors were used to determine the parameters of interelectronic repulsion (Racah parameters $B$ and $C$ ) and the one-electron $5 \times 5$ LF matrix in a least-square sense, 4) these parameters were used as input for the conventional LF program, thereby enabling calculation of all the multiplets. The DFT part of LF-DFT, that is, AOC and SD calculations, were performed on OPBE-optimized geometries by using the OPBE functional. Experimentally obtained SOC constants for free-metal ions, ${ }^{[28]}$ reduced by an orbital reduction factor, ${ }^{[13]}$ were used for calculations of the MAEs.

The TZP basis set was used for all atoms in all DFT calculations. Optimization of the investigated complexes, and AOC and SD calculations were realized by using the Amsterdam density functional (ADF) program package. ${ }^{[33]}$

\section{Acknowledgements}

This work was financially supported by the Serbian Ministry of Education, Science and Technological Development (Grant No. 172035), the Swiss National Science Foundation, and the Spanish Ministerio of Economía y Competitividad under Project FIS2012-37549-C05-4. P.G.-F. was supported by the Ramon y Cajal programme with grant RYC-2013-12515. The support of COST Action CM1305 "Explicit Control Over Spin-states in Technology and Biochemistry (ECOSTBio)" is also acknowledged. Part of this work was also supported by the COST Action CM1006 and "EUFEN" financed by the Swiss State Secretariat for Research and Innovation.

Keywords: density functional calculations - Jahn-Teller distortion - ligand-field effects - magnetic properties scorpionate complexes

[1] a) P. W. Anderson, Phys. Rev. 1959, 115, 2-13; b) P. J. Hay, J. C. Thibeault R. Hoffmann, J. Am. Chem. Soc. 1975, 97, 4884-4899; c) G. Rajaraman, F. Totti, A. Bencini, A. Caneschi, R. Sessoli, D. Gatteschi, Dalton Trans. 2009 3153-3161; d) A. Bencini, M. Casarin, D. Forrer, L. Franco, F. Garau, N. Masciocchi, L. Pandolfo, C. Pettinari, M. Ruzzi, A. Vittadini, Inorg. Chem. 2009, 48, 4044-4051; e) A. Bencini, F. Totti, J. Chem. Theory Comput. 2008, 4, 144-154; f) A. Bencini, F. Totti, Int. J. Quantum Chem. 2005, $101,819-825$; g) C. Duboc, D. Ganyushin, K. Sivalingam, M.-N. L. Collomb, F. Neese, J. Phys. Chem. A 2010, 114, 10750-10758; h) D. A. Pantazis, V. Krewald, M. Orio, F. Neese, Dalton Trans. 2010, 39, 4959-4967; i) M. Orio, D. A. Pantazis, T. Petrenko, F. Neese, Inorg. Chem. 2009, 48, $7251-7260$; j) E. Ruiz, A. Rodríguez-Fortea, J. Cano, S. Alvarez, P. Alemany, J. Comput. Chem. 2003, 24, 982-989; k) O. Kahn, Molecular Magnetism, Wiley-VCH, New York, 1993, p. 396.

[2] a) R. Sessoli, H. L. Tsai, A. R. Schake, S. Wang, J. B. Vincent, K. Folting, D. Gatteschi, G. Christou, D. N. Hendrickson, J. Am. Chem. Soc. 1993, 115 $1804-1816$; b) R. Sessoli, D. Gatteschi, A. Caneschi, M. A. Novak, Nature 1993, 365, $141-143$.

[3] a) D. Gatteschi, R. Sessoli, J. Villain, Molecular Nanomagnets, Oxford University Press, Oxford, 2006, p.416; b) R. Winpenny, Single-Molecule Magnets and Related Phenomena, Springer, Berlin, Heidelberg 2006, p. 262.

[4] C. J. Calzado, J. Cabrero, J. P. Malrieu, R. Caballol, J. Chem. Phys. 2002, $116,2728-2747$

[5] a) O. Waldmann, Inorg. Chem. 2007, 46, 10035-10037; b) F. Neese, D. A Pantazis, Faraday Discuss. 2011, 148, 229-238; c) S. K. Singh, G. Rajaraman, Chem. Eur. J. 2014, 20, 5214-5218.
[6] J. Cirera, E. Ruiz, S. Alvarez, F. Neese, J. Kortus, Chem. Eur. J. 2009, 15, $4078-4087$.

[7] a) S. Ye, F. Neese, J. Chem. Theory Comput. 2012, 8, 2344-2351; b) K. S. Pedersen, J. Bendix, R. Clerac, Chem. Commun. 2014, 50, 4396-4415; c) S. K. Singh, T. Gupta, P. Badkur, G. Rajaraman, Chem. Eur. J. 2014, 20, 10305-10313; d) S. Gómez-Coca, E. Cremades, N. Aliaga-Alcalde, E. Ruiz, Inorg. Chem. 2013, 52, 676-678; e) S. Gomez-Coca, E. Cremades, N. Aliaga-Alcalde, E. Ruiz, J. Am. Chem. Soc. 2013, 135, 7010-7018.

[8] a) H. Kurzen, L. Bovigny, C. Bulloni, C. Daul, Chem. Phys. Lett. 2013, 574, 129-132; b) F. Neese, J. Am. Chem. Soc. 2006, 128, 10213-10222; c) T. Rajeshkumar, G. Rajaraman, Chem. Commun. 2012, 48, 7856-7858; d) S. K. Singh, N. K. Tibrewal, G. Rajaraman, Dalton Trans. 2011, 40, 10897-10906; e) L. F. Chibotaru, L. Ungur, J. Chem. Phys. 2012, 137, 064112; f) L. F. Chibotaru, A. Ceulemans, H. Bolvin, Phys. Rev. Lett. 2008, 101, 033003.

[9] W. Kohn, L. J. Sham, Phys. Rev. 1965, 140, A1133-A1138.

[10] a) M. Atanasov, C. Rauzy, P. Baettig, C. Daul, Int. J. Quantum Chem. 2005, 102, 119-131; b) M. Atanasov, C. A. Daul and C. Rauzy in A DFT Based Ligand Field Theory, Vol. 106 (Eds.: D. Mingos and T. Schönherr), Springer, Berlin, Heidelberg, 2004, pp.97-125; c) M. Atanasov, C. A. Daul, C. Rauzy, Chem. Phys. Lett. 2003, 367, 737-746; d) F. Senn, L. Helm, A. Borel, C. A. Daul, C. R. Chim. 2012, 15, 250-254; e) M. Atanasov, C. A. Daul, M.-M. Rohmer, T. Venkatachalam, Chem. Phys. Lett. 2006, 427, 449-454; f) L. Petit, A. Borel, C. Daul, P. Maldivi, C. Adamo, Inorg. Chem. 2006, 45, 7382-7388; g) M. Atanasov, P. Comba, C. A. Daul, Inorg. Chem. 2008, 47, 2449-2463; h) A. Trueba, P. Garcia-Fernandez, F. Senn, C. A. Daul, J. A. Aramburu, M. T. Barriuso, M. Moreno, Phys. Rev. B 2010, 81, 075107; i) P. Garcia-Fernandez, F. Senn, C. A. Daul, J. A. Aramburu, M. T. Barriuso, M. Moreno, Phys. Chem. Chem. Phys. 2009, 11, 7545-7548.

[11] a) T. Ziegler, A. Rauk, Theor. Chim. Acta 1977, 46, 1-10; b) C. Daul, Int. J. Quantum Chem. 1994, 52, 867-877.

[12] a) A. Trueba, P. Garcia-Fernandez, J. M. García-Lastra, J. A. Aramburu, M. T. Barriuso, M. Moreno, J. Phys. Chem. A 2011, 115, 1423-1432; b) A. Borel, L. Helm, C. A. E. Daul, Chem. Phys. Lett. 2004, 383, 584-591; c) M. Gruden-Pavlović, M. Peric, M. Zlatar, P. Garcia-Fernandez, Chem. Sci. 2014, 5, 1453-1462.

[13] M. Atanasov, E. Jan Baerends, P. Baettig, R. Bruyndonckx, C. Daul, C. Rauzy, M. Zbiri, Chem. Phys. Lett. 2004, 399, 433-439.

[14] M. Atanasov, P. Comba, C. A. Daul, A. Hauser, J. Phys. Chem. A 2007, 111, 9145-9163.

[15] M. Atanasov, C. A. Daul, Chem. Phys. Lett. 2003, 381, 584-591.

[16] a) F. Senn, C. A. Daul, Theochem. J. Mol. Struct. 2010, 954, 105-108; b) F. Senn, M. Zlatar, M. Gruden-Pavlovic, C. Daul, Monatsh. Chem. 2011, 142, $593-597$.

[17] a) M. Zbiri, C. A. Daul, T. A. Wesolowski, J. Chem. Theory Comput. 2006, 2, 1106-1111; b) M. Zbiri, M. Atanasov, C. Daul, J. M. Garcia-Lastra, T. A. Wesolowski, Chem. Phys. Lett. 2004, 397, 441-446; c) M. Atanasov, C. Daul, H. U. Güdel, T. A. Wesolowski, M. Zbiri, Inorg. Chem. 2005, 44, 2954- 2963.

[18] a) H. Ramanantoanina, W. Urland, A. García-Fuente, F. Cimpoesu, C. Daul, Chem. Phys. Lett. 2013, 588, 260-266; b) H. Ramanantoanina, W. Urland, F. Cimpoesu, C. Daul, Phys. Chem. Chem. Phys. 2013, 15, 13902 13910.

[19] M. Atanasov, C. A. Daul, C. R. Chim. 2005, 8, 1421-1433.

[20] a) J. Krzystek, D. C. Swenson, S. A. Zvyagin, D. Smirnov, A. Ozarowski, J. Telser, J. Am. Chem. Soc. 2010, 132, 5241-5253; b) P. J. Desrochers, J. Telser, S. A. Zvyagin, A. Ozarowski, J. Krzystek, D. A. Vicic, Inorg. Chem. 2006, 45, 8930-8941.

[21] a) M. Atanasov, D. Ganyushin, D. A. Pantazis, K. Sivalingam, F. Neese, Inorg. Chem. 2011, 50, 7460-7477; b) B. R. McGarvey, J. Telser, Inorg. Chem. 2012, 51, 6000-6010.

[22] a) I. Bersuker, The Jahn-Teller Effect, Cambridge University Press, Cambridge, 2006, p. 634; b) M. Zlatar, C.-W. Schläpfer, E. P. Fowe, C. A. Daul, Pure Appl. Chem. 2009, 81, 1397-1411; c) M. Zlatar, M. Gruden-Pavlović, C.-W. Schläpfer, C. Daul, Theochem. J. Mol. Struct. 2010, 954, 86-93; d) M. Gruden-Pavlović, M. Zlatar, C.-W. Schläpfer, C. Daul, Theochem. J. Mol. Struct. 2010, 954, 80-85.

[23] a) R. Maurice, R. Bastardis, C. d. Graaf, N. Suaud, T. Mallah, N. Guihéry, J. Chem. Theory Comput. 2009, 5, 2977-2984; b) R. Maurice, C. de Graaf, N. Guihéry, J. Chem. Phys. 2010, 133. 
[24] A. V. Palii, J. M. Clemente-Juan, E. Coronado, S. I. Klokishner, S. M. Ostrovsky, O. S. Reu, Inorg. Chem. 2010, 49, 8073-8077.

[25] M. Atanasov, J. M. Zadrozny, J. R. Long, F. Neese, Chem. Sci. 2013, 4, $139-156$.

[26] S. Ye, F. Neese, A. Ozarowski, D. Smirnov, J. Krzystek, J. Telser, J.-H. Liao, C.-H. Hung, W.-C. Chu, Y.-F. Tsai, R.-C. Wang, K.-Y. Chen, H.-F. Hsu, Inorg. Chem. 2009, 48, 977-988.

[27] CASSCF/NEVPT2 calculations were performed with the ORCA program package (F. Neese, An ab initio, DFT and semiempirical electronic structure package, version 3.0.2., Max-Planck-Institute für Bioanorganische Chemie, Mulheim, Germany, 2009.). Quasidegenerate perturbation theory was used for SOC. The ZORA approximation was employed with the ZORA-TZVP basis set for all atoms. $\operatorname{CAS}(2,5)$ was used for the vanadium complex and $\operatorname{CAS}(3,5)$ for the chromium complex. The orbitals were optimized by the average of 10 triplets and 15 singlets for the vanadium complex and 10 quartets and 40 doublets for the chromium complex. The RI approximation was used to speed up the calculations along with the TZV/C auxiliary basis sets. The state energies used in quasidegenerate perturbation theory are corrected to second order by NEVPT2 calculations.

[28] J. S. Griffith, The Theory of Transition-Metal Ions, University Press, Cambridge, Cambridge (UK), 1964, p. 455.

[29] a) W. H. Harman, T. D. Harris, D. E. Freedman, H. Fong, A. Chang, J. D. Rinehart, A. Ozarowski, M. T. Sougrati, F. Grandjean, G. J. Long, J. R. Long C. J. Chang, J. Am. Chem. Soc. 2010, 132, 18115-18126; b) D. E. Freed- man, W. H. Harman, T. D. Harris, G. J. Long, C. J. Chang, J. R. Long, J. Am. Chem. Soc. 2010, 132, 1224-1225.

[30] N. C. Handy, A. J. Cohen, Mol. Phys. 2001, 99, 403-412.

[31] J. P. Perdew, K. Burke, M. Ernzerhof, Phys. Rev. Lett. 1996, 77, $3865-$ 3868.

[32] M. Swart, A. W. Ehlers, K. Lammertsma, Mol. Phys. 2004, 102, 24672474.

[33] Amsterdam density functional (ADF) program package, E. J. Baerends, J. Autschbach, A. Bérces, J. A. Berger, F. M. Bickelhaupt, C. Bo, P. L. de Boeij, P. M. Boerrigter, L. Cavallo, D. P. Chong, L. Deng, R. M. Dickson, D. E. Ellis, M. van Faassen, L. Fan, T. H. Fischer, C. Fonseca Guerra, S. J. A. van Gisbergen, J. A. Groeneveld, O. V. Gritsenko, M. Grüning, F. E. Harris, P. van den Hoek, C. R. Jacob, H. Jacobsen, L. Jensen, E. S. Kadantsev, G. van Kessel, R. Klooster, F. Kootstra, E. van Lenthe, D. A. McCormack, A. Michalak, J. Neugebauer, V. P. Nicu, V. P. Osinga, S. Patchkovskii, P. H. T. Philipsen, D. Post, C. C. Pye, W. Ravenek, P. Romaniello, P. Ros, P. R. T. Schipper, G. Schreckenbach, J. G. Snijders, M. Solà, M. Swart, D. Swerhone, G. teVelde, P. Vernooijs, L. Versluis, L. Visscher, O. Visser, F. Wang, T. A. Wesolowski, E. M. van Wezenbeek, G. Wiesenekker, S. K. Wolff, T. K. Woo, A. L. Yakovlev, T. Ziegler, SCM, Amsterdam, 2013. 